\title{
Branch Retinal Vein Occlusion in a Patient With Familial Hyperlipidemia
}

Leonid Skorin, Jr, DO, OD, MS ${ }^{1,2}$. Lucas J. Asfeld, OD

A 57-year-old-man presented to the eye clinic with painless decreased vision of unknown onset that he attributed to needing new glasses.

\section{History}

His medical history was significant for familial hyperlipidemia, for which he was taking atorvastatin, $5 \mathrm{mg}$, daily. In addition, he was taking lisinopril, 10 $\mathrm{mg}$, daily for the treatment of hypertension. His family history included carotid artery disease and a brother who died at age 48 years from a myocardial infarction.

Further review of the patient's medical history revealed that he had poorly controlled hyperlipidemia, even with taking atorvastatin. A lipid panel, conducted 6 months prior to his visit to the eye clinic, revealed a total cholesterol level of $349 \mathrm{mg} / \mathrm{dL}$ (reference range, < $200 \mathrm{mg} / \mathrm{dL}$ ), a triglyceride level of 152 $\mathrm{mg} / \mathrm{dL}$ (reference range, $<150 \mathrm{mg} / \mathrm{dL}$ ), a high-density lipoprotein (HDL) level of $73 \mathrm{mg} / \mathrm{dL}$ (reference range, $\geq 50 \mathrm{mg}$ / $d L)$, a low-density lipoprotein (LDL) level of $246 \mathrm{mg} / \mathrm{dL}$ (reference range, $<100 \mathrm{mg} / \mathrm{dL}$ ), and a non-HDL level of $276 \mathrm{mg} / \mathrm{dL}$ (reference range, < $130 \mathrm{mg}$ / $\mathrm{dL}$ ). His blood pressure at that time was $128 / 80 \mathrm{mmHg}$. Due to his elevated lipid panel results, the patient's primary care physician recommended a cardiology consultation, which the patient declined.

\section{Physical Examination}

At the eye clinic visit, the patient's best corrected visual acuities were 20/100 in the right eye and 20/20 in the left eye. The intraocular pressure was $16 \mathrm{mmHg}$ in each eye. Both eyes showed normal pupil function with no relative afferent pupillary defect and normal confrontation visual field testing.

Examination of the fundus revealed dense superior dot/blot and flame-shaped hemorrhages, several small cotton wool spots, and hard exudates near the macula (Figures 1 and 2). These findings were indicative of a superior branch retinal vein

\section{AFFILIATIONS:}

'Department of Surgery, Community Division of Ophthalmology, Mayo Clinic Health System Albert Lea, Minnesota

${ }^{2}$ Assistant Professor, Ophthalmology Department, Mayo Clinic College of Medicine, Rochester, Minnesota

${ }^{3}$ Recent Graduate, Pacific University College of Optometry, Forest Grove, Oregon

\section{CITATION:}

Skorin Jr L, Asfeld LJ. Branch retinal vein occlusion in a patient with familial hyperlipidemia. Consultant. Published online May 26, 2021. doi:10.25270/con.2021.05.00013

Received January 13, 2021. Accepted January 29, 2021.

\section{DISCLOSURES:}

The authors report no relevant financial relationships.

\section{CORRESPONDENCE:}

Leonid Skorin, Jr, DO, OD, MS, 404 West Fountain Street, Albert Lea, MN 56007 (skorin.leonid@mayo. edu)

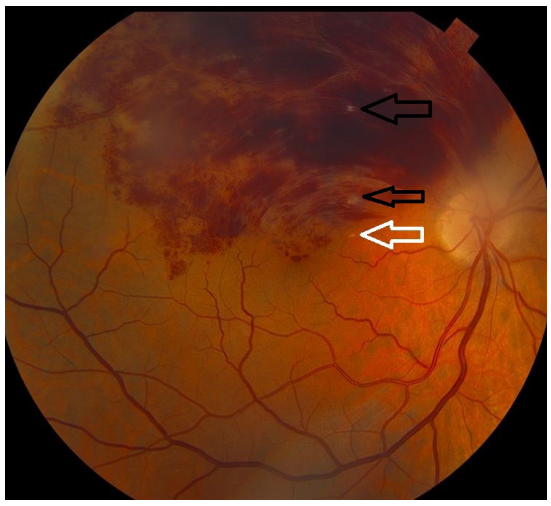

Figure 1. Image of the fundus of the right eye with superior temporal BRVO showed dense superior dot/blot and flame-shaped hemorrhages, small cotton wool spots (black arrows), and hard exudates near the macula (white arrow).

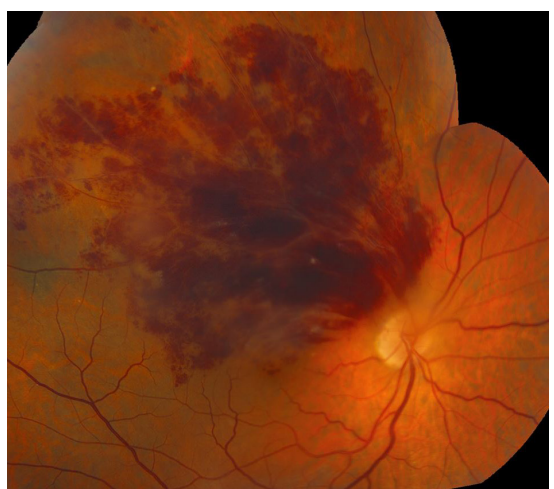

Figure 2. Image of the montage fundus of the right eye showed the full extent of the retinal hemorrhages in the peripheral fundus.

occlusion (BRVO) of the right eye.

Results of an optical coherence tomography (OCT) scan of the right eye showed intraretinal fluid of the superior macula (Figure 3), a major cause of reduced vision in patients with retinal vein occlusion. 'Normal macular thickness 


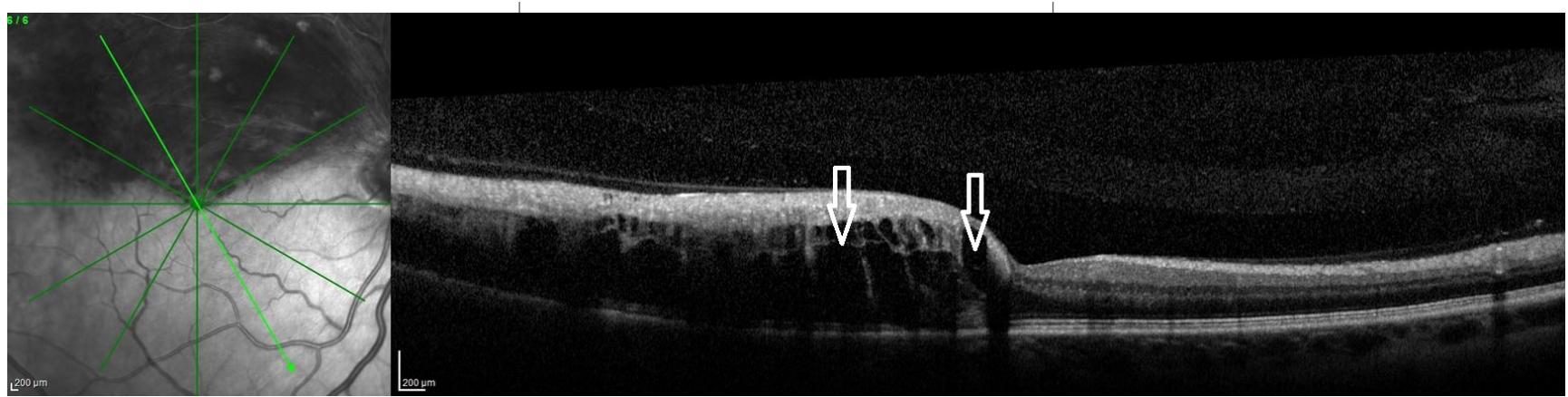

Figure 3. OCT scans of the right eye showed macular intraretinal fluid (edema) of the superior macula (arrows). Increased thickness of the entire superior macula and surrounding retina can also be seen because of extensive hemorrhaging.

in a healthy eye averages 270.2 microns. $^{2}$ Our patient's central macular thickness on OCT scan was 561 microns in the right eye (Figure 4). OCT is a noninvasive imaging technique that uses light waves to produce high-resolution cross-sectional images of the retina. ${ }^{3}$

Fluorescein angiography was also performed to assess the retinal vascular integrity, results of which showed a large area of blockage and nonperfusion along the superior retinal vascular arcade (Figure 5). Punctate hyperfluorescence was also found at the inferior border near the macula, indicating leakage from the surrounding blood vessels (Figure 6). Fluorescein angiography is performed via intravenous injection of sodium fluorescein into a patient's arm. ${ }^{4}$ The dye travels through the systemic circulation and eventually reaches the eye. Fundus images are then captured as the fluorescent dye travels through the retinal vasculature. This imaging technique is used to identify retinal ischemia, microaneurysms, neovascularization, and macular edema.

\section{Discussion}

A BRVO results from the blockage of blood flow leaving the retinal vasculature, most often caused by compression of a retinal vein by a retinal artery. This compression results in turbulent blood flow, causing endothelial damage of the vessel and thrombus formation. Common fundus examination findings include large wedge-shaped retinal hemorrhages, cot-
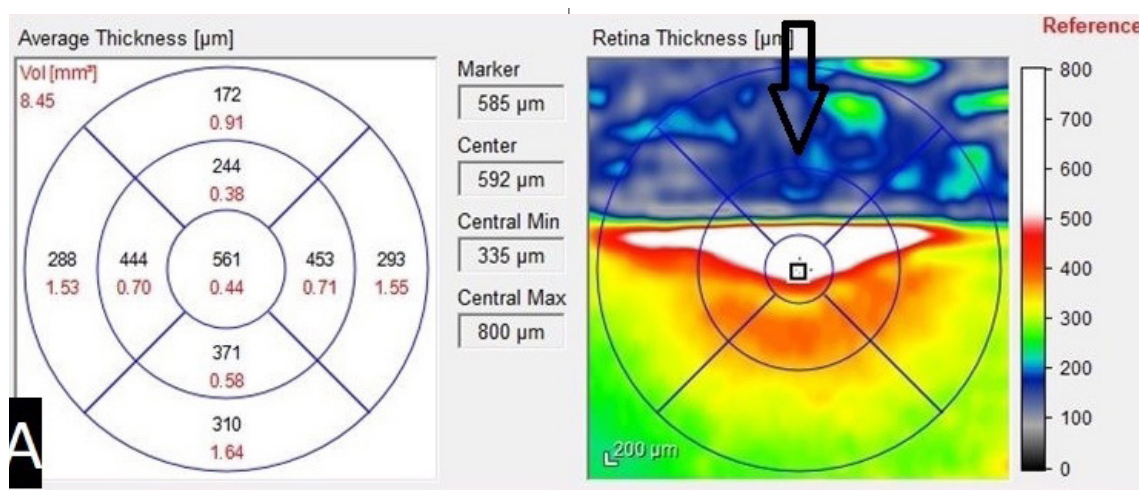

Figure 4. An OCT heat map and numeric grid showed the central macular thickness (561 microns) of the right eye pretreatment. The arrow indicates an area of false data caused by significant retinal thickening and hemorrhage.

ton wool spots caused by ischemia, attenuated arterioles, dilated tortuous veins, and retinal edema. These findings usually occur at retinal arteriovenous crossings in the inferior or, more commonly, superior temporal areas..$^{5,6}$ Optic disc hyperemia and swelling may also be seen, but these were not present in our patient (Figure 7).

Retinal vein occlusions (RVOs) can be categorized as central, hemicentral, or branch depending on the site of occlusion. ${ }^{7}$ Central RVOs involve the entire retina due to a blockage in the central vein posterior to the optic nerve head. ${ }^{6,7}$ Hemicentral RVOs involve either the superior or inferior retinal hemisphere due to an occlusion just distal to the bifurcation of the central vein. ${ }^{6,7}$ BRVOs typical occur when an occlusion of the smaller tributary veins in the retina is present, ${ }^{6,7}$ RVOs can be categorized as nonischemic or ischemic, the latter of which includes large areas of retinal capillary vessel nonperfusion. This capillary nonperfusion can result in abnormal and leaky blood vessel growth (neovascularization) of the optic disc, retina, and iris. Neovascularization of the iris, also known as iris rubeosis, can cause secondary glaucoma caused by abnormal growth of blood vessels in the drainage system of the eye known as the trabecular meshwork. ${ }^{7}$ This blockage of the trabecular meshwork can cause inhibition of aqueous fluid outflow, resulting in elevated intraocular pressure and damage to the optic nerve. ${ }^{7}$ For this reason, patients need to be monitored closely by an eye care professional following any RVO event, since even nonischemic RVOs can convert to ischemic RVOs. Patients who develop ischemia and neovascularization tend to have worse prognosis. ${ }^{5,6}$

Symptoms of an RVO include sud- 


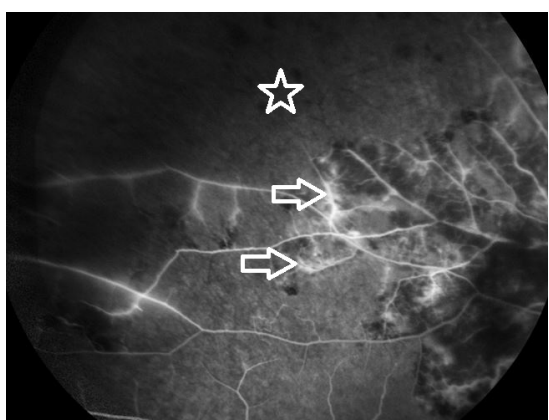

Figure 5. Fluorescein angiography showed nonperfusion (star) and leaking retinal vessels (arrows).

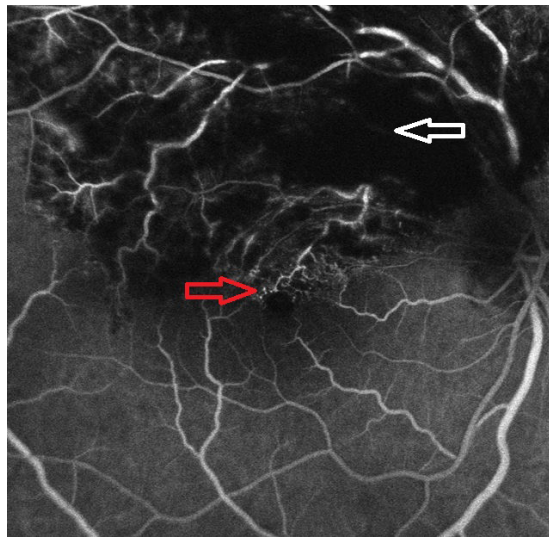

Figure 6. Fluorescein angiography of the right eye showed blockage (white arrow) caused by retinal hemorrhage in the superior hemisphere. Punctate hyperfluorescence is seen at the inferior border near the macula, indicating leakage from the surrounding blood vessels (red arrow).

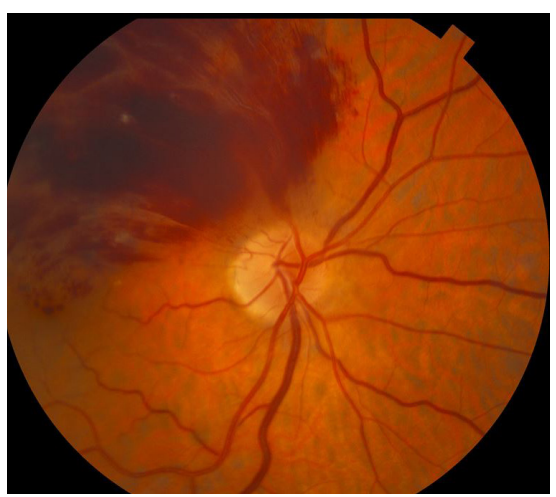

Figure 7. Image of the fundus of the right eye showed no edema or swelling of the optic disc. den, unilateral, painless vision loss that can vary depending on the severity of retinal involvement and the presence of macular edema. Initial vision loss is typically caused by blood and exudates in the macular area that usually resolve in weeks to months with minimal permanent damage to vision. Severe and permanent vision loss occurs primarily from macular ischemia, chronic macular edema, and complications resulting from iris neovascularization. ${ }^{5,8}$

Treatment of edema, retinal or iris neovascularization, vitreous hemorrhage, and secondary tractional retinal detachment associated with RVOs has typically included panretinal, macular grid, and scatter laser photocoagulation. ${ }^{5}$ However, the newest treatment of macular edema in RVOs now includes the use of intravitreal injections.

Intravitreal antivascular endothelial growth factor (anti-VEGF) injections are now typically first-line treatment. ${ }^{6}$ Common agents include ranibizumab, aflibercept, and bevacizumab. ${ }^{6}$ Retinal ischemia following an RVO causes a large and immediate release of VEGF, which stimulates neovascularization in the retina and iris. Anti-VEGF injections inhibit the effect of VEGF and prevent formation of neovascularization and retinal edema. For this reason, anti-VEGF agents are most effective when early intervention is initiated. ${ }^{9}$ One analysis also showed a linear relationship between mean improvement in letters gained on a visual acuity chart and mean number of anti-VEGF injections at 6 months and 1 year after initiation of treatment. ${ }^{10}$ This study also showed that those with worse initial entering visual acuities saw greater improvements in vision after treatment. $^{10}$

Intravitreal steroid injections such as dissolvable dexamethasone implants or intravitreal triamcinolone, have also been used to treat RVOs. ${ }^{6}$ They have been especially beneficial in patients with macular edema. ${ }^{11,12}$ This is thought to be because of the steroids' inhibitory effect on inflammatory mediators and an overall reduction in vascular permeability. Both the release of inflammatory mediators and vascular permeability are responses to ischemia and result in macular edema. However, use of intravitreal steroids can result in adverse events such as elevated intraocular pressure, cataract formation, and rarely endophthalmitis. ${ }^{11,12}$ For this reason, intravitreal steroid treatment is typically reserved as a second-tier treatment when other treatments have failed.

Strong risk factors for RVOs include hyperlipidemia, hypertension, and diabetes. ${ }^{5,13}$ Other risk factors include increasing age, arteriosclerosis, vascular cerebral stroke, blood hyperviscosity, and thrombophilia. ${ }^{5,13}$ In our case, the patient had a history of hypertension and uncontrolled familial hyperlipidemia. A meta-analysis of 2915 patients with RVOs from 21 observational studies over a 20year period was conducted. ${ }^{14}$ Its findings revealed that the risk of RVOs was more than 3.5 times higher in patients with systemic hypertension and about 2.5 times higher in patients with hyperlipidemia. ${ }^{14}$ Hyperlipidemia is an imbalance of HDL and LDL. ${ }^{15}$ Although RVOs were not specifically addressed, a report from the American Heart Association showed that reducing LDL levels resulted in lowered rates of major vascular events. ${ }^{16}$ Therefore, mainstay treatment typically consists of statins, which are used to lower LDL levels. However, many patients with hyperlipidemia, and especially those with the familial type, may not achieve adequate LDL lowering with statin drugs alone. ${ }^{15}$ Because of his history of familial hyperlipidemia, our patient fell into this category.

\section{Patient outcome}

The patient was started on bevacizumab intravitreal anti-VEGF injections, as indicated for the treatment of macular edema in patients with RVO.' These injections occurred every 4 weeks for a series of 4 injections in the right eye. At the conclusion of his first intravitreal injection series, the patient's vision improved in the right eye to 20/20. An examination of the fundus showed resolving hemorrhages 

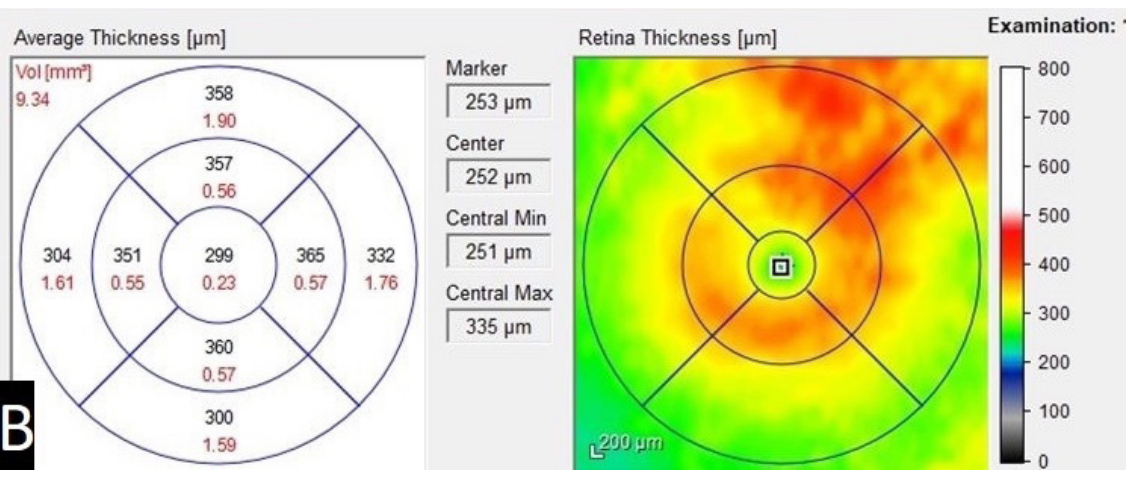

Figure 8. A follow-up OCT heat map and numeric grid showed the central macular thickness (299 microns) of the right eye post-treatment.

and exudates. A repeat OCT showed reduction in macular thickness to 299 microns (Figure 8) and almost complete resolution of the macular intraretinal fluid (Figure 9). Because he had a good response to the intravitreal injections, the patient was scheduled for another series of 5 bevacizumab injections every 4 to 5 weeks in the right eye.

The patient was seen by a cardiologist one month after presenting to the eye clinic. At this visit, the patient was started on evolocumab, $140 \mathrm{mg}$, subcutaneous injections every 14 days and continued with atorvastatin, $5 \mathrm{mg}$, daily. Evolocumab is a fully human monoclonal antibody used to lower LDL cholesterol levels by inhibiting proprotein convertase subtilisin/kexin type $9 .^{17}$ The patient's blood pressure was elevated to $158 / 90$ $\mathrm{mmHg}$ at this visit, so his prescription for lisinopril was increased to $20 \mathrm{mg}$ daily. One month after this visit, his blood pressure was found to be better controlled at $128 / 84 \mathrm{mmHg}$ with the increase in the lisinopril. Two months after starting evolocumab injections, the patient's total cholesterol level improved to $183 \mathrm{mg} / \mathrm{dL}$, triglyceride level improved to $147 \mathrm{mg} / \mathrm{dL}$, HDL level remained relatively stable at 68 $\mathrm{mg} / \mathrm{dL}$, LDL level improved to $86 \mathrm{mg} / \mathrm{dL}$, and non-HDL level improved to $115 \mathrm{mg} /$ $\mathrm{dL}$. These results showed the initial lack of the patient's response to atorvastatin alone for the treatment of familial hyperlipidemia. However, better control was found with the addition of evolocumab subcutaneous injections.

\section{Conclusion}

This case shows that although RVOs can have severe visual consequences, there are treatment options available that can significantly improve visual outcomes. For this reason, it is imperative for an ophthalmologist referral. This will allow early initiation of treatment to preserve the patient's vision. It is also critical to have continued care between both primary care physicians and eye care professionals to manage both the systemic disease and associated ocular complications. Better control of this patient's lipids and blood pressure should help prevent additional retinal vascular events.

\section{References}

1. Lashay A, Riazi-Esfahani H, Mirghorbani M, Yaseri M. Intravitreal medications for retinal vein occlusion: systematic review and meta-analysis. $J$ Ophthalmic Vis Res. 2019;14(3):336-366. https://doi. org/10.18502/jovr.v14i3.4791

2. Grover S, Murthy RK, Brar VS, Chalam KV. Normative data for macular thickness by high-definition spectral-domain optical coherence tomography (spectralis). Am J Ophthalmol. 2009;148(2):266-271. https:// doi.org/10.1016/j.ajo.2009.03.006

3. Podoleanu AG. Optical coherence tomography. J Microsc. 2012;247(3):209-219. https:// doi.org/10.1111/j.1365-2818.2012.03619.x

4. Bennett TJ, Quillen DA, Coronica R. Fundamentals of fluorescein angiography. Insight. 2016;41(1):5-11. http://eye-pix.com/wp-content/uploads/Bennett-ASORN-2016.pdf

5. Yau JW, Lee P, Wong TY, Best J, Jenkins A. Retinal vein occlusion: an approach to diagnosis, systemic risk factors and management. Intern Med J. 2008;38(12):904910. https://doi.org/10.1111/j.14455994.2008.01720.x

6. Handza JM. Retinal venous occlusive disease. In: Onofrey BE, Skorin Jr L, Holdeman NR, eds. Ocular Therapeutics Handbook: A Clinical Manual. 4th ed. Wolters Kluwer; 2019:536-540.

7. Ip $M$, Hendrick A. Retinal vein occlusion review. Asia Pac J Ophthalmol (Phila).

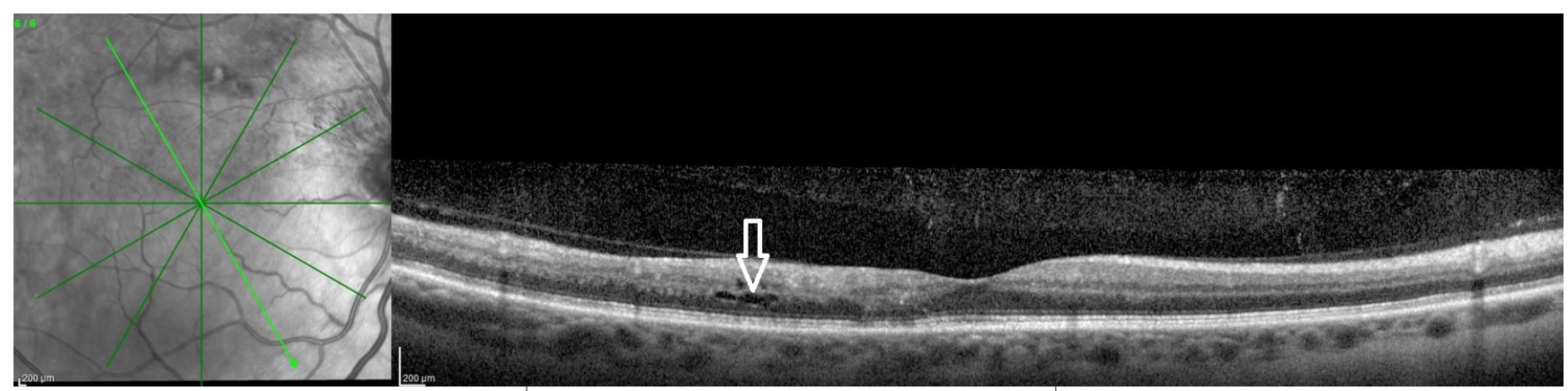

Figure 9. A follow-up OCT of the right eye showed almost complete resolution of the macular intraretinal fluid. White arrow points to residual fluid. 
2018;7(1):40-45. https://doi.org/10.22608/ apo.2017442

8. Skorin L Jr, Lauermann T. Retinal vein occlusions: diagnosis and management. Consultant. 2020;60(10):3-5,12,16. doi:10.25270/ con.2020.05.00016

9. Noma H, Yasuda K, Shimura M. Cytokines and the pathogenesis of macular edema in branch retinal vein occlusion. $J$ Ophthalmol. 2019;2019:5185128. https://doi. org/10.1155/2019/5185128

10. Ciulla T, Pollack JS, Williams DF. Visual acuity outcomes and anti-VEGF therapy intensity in macular oedema due to retinal vein occlusion: a real-world analysis of 15613 patient eyes. Br J Ophthalmol. 2020;bjophthalmol-2020-317337. https:// doi.org/10.1136/bjophthalmol-2020-317337

11. Haller JA, Bandello F, Belfort R Jr, et al; OZURDEX GENEVA Study Group. Randomized, sham-controlled trial of dexamethasone intravitreal implant in patients with macular edema due to retinal vein occlusion. Ophthalmology. 2010;117(6):11341146.e3. https://doi.org/10.1016/j.ophtha.2010.03.032

12. Scott IU, Ip MS, VanVeldhuisen PC, et al. $A$ randomized trial comparing the efficacy and safety of intravitreal triamcinolone with standard care to treat vision loss associated with macular Edema secondary to branch retinal vein occlusion: the Standard Care vs Corticosteroid for Retinal Vein Occlusion (SCORE) study report 6. Arch Ophthalmol. 2009;127(9):1115-1128. https://doi. org/10.1001/archophthalmol.2009.233

13. Kolar P. Risk factors for central and branch retinal vein occlusion: a meta-analysis of published clinical data. $J$ Ophthalmol. 2014;2014:724780. https://doi. org/10.1155/2014/724780

14. O'Mahoney PR, Wong DT, Ray JG. Retinal vein occlusion and traditional risk factors for atherosclerosis. Arch Ophthalmol. 2008;126(5):692-699. https://doi. org/10.1001/archopht.126.5.692

15. Karr S. Epidemiology and management of hyperlipidemia. Am J Manag Care. 2017;23(9 Suppl):S139-S148. https://www.ajmc. com/view/epidemiology-and-management-of-hyperlipidemia-article

16. Virani SS, Alonso A, Benjamin EJ, et al;
American Heart Association Council on Epidemiology and Prevention Statistics Committee and Stroke Statistics Subcommittee. Heart disease and stroke statistics-2020 update: a report from the American Heart Association. Circulation. 2020;141(9):e139-e596. https://doi. org/10.1161/cir.0000000000000757

17. Kasichayanula S, Grover A, Emery MG, et al. Clinical pharmacokinetics and pharmacodynamics of evolocumab, a PCSK9 inhibitor. Clin Pharmacokinet. 2018;57(7):769-779. https://doi.org/10.1007/s40262-017-0620712. 